\title{
Reflections on the Integration of Short Videos Into High School Art Appreciation Teaching Under the Background of Covid-19 Epidemic
}

\author{
${ }^{1}$ JiMei University, Jimei, Xiamen, Fujian, China \\ ${ }^{2}$ JiMei University, Jimei, Xiamen, Fujian, China \\ 1140751800@qq.com \\ b*519721193@qq.com
}

Qiduan $\mathrm{Chen}^{1, \mathrm{a}}$,Xueying $\mathrm{Yu}^{2, \mathrm{~b}^{*}}$

\begin{abstract}
Under the influence of the Covid-19 epidemic in 2020, the Ministry of Education has called for the adoption of an "Internet + Education Model" to achieve the goal of "keeping educating and learning during class suspension". In this context, to better cultivate students' ability to appreciate art, a short video is used to integrate art appreciation units of different topics into teaching high school art appreciation. We are thereby able to analyze the differences between use of short videos, traditional teaching and other online teaching applications. In addition, this paper proposes a new mode of applying short videos to online teaching, which provides a reference for the effective implementation of online teaching. The study shows that the use of short videos to comprehensively improve the quality of teaching art appreciation in high schools can help students effectively apply the knowledge of different units they have learned, transform scattered art appreciation knowledge into diversified and systematic art appreciation abilities, and promote students' enthusiasm for art appreciation; in actual teaching, overemphasis of traditional teaching methods cannot fully meet the needs of current students. The successful integration of short videos into teaching requires vigorous cooperation of students, teachers' effective control and design of the teaching process, more precise handling of teaching activities, and professional teaching methods that meet the needs of students. Only in this way can the "Internet + Education Model" be implemented, the quality of teaching be improved all around, and students' deep growth and interest in learning in art appreciation be promoted.
\end{abstract}

Keywords: Short Videos, Art Appreciation, Covid-19, Online Teaching

\section{新冠疫情背景下短视频融入高中美术鉴赏教学之思考}

陈其端 $^{1, a}$ ，余雪莹 ${ }^{2, b *}$

\footnotetext{
'集美大学, 集美, 厦门, 福建, 中国

${ }^{2}$ 集美大学, 集美, 厦门, 福建, 中国

a1140751800@qq.com

b*519721193@qq.com
}

\section{摘要}

2020 年新冠疫情影响下，教育部号召采用 “互联网+教育模式”，“停课不停教、停课不停学”，在此背景下 为了更好地培养学生美术鉴赏的能力,研究者利用短视频设计以不同主题的美术鉴赏单元融入高中美术鉴赏教 学为例, 分析短视频教学与一般的传统教学的差别. 提出短视频应用于在线教学的新模式. 研究发现利用短视频 全面提升高中美术鉴赏教学质量能够助力学生有效地应用自己所学习的不同单元知识,将零散的美术鉴赏知识 转变为多元化以及系统化, 促使学生形成对美术鉴赏学习的积极性; 在开展实际教学活动的过程中, 如果教师 过分强调使用传统教学方法, 显然无法充分贴合现下学生群体的需要. 成功的短视频融入教学活动不仅需要学 
生群体的大力配合, 更需要教师对教学流程进行有效管控设计, 对课堂教学活动予以更为精准的处理, 提取最 符合学生群体的专业教学方法, 只有这样才能落实互联网+教育模式全面提高教学质量, 助力学生在美术鉴赏 素养的深度成长与学习兴趣。

关键词: 短视频教学; 新冠疫情; 美术鉴赏

\section{1. 前言}

根据第 46 次中国互联网发展统计报告显示, 截 至 2020 年 6 月, 我国网络视频（含短视频）用户规 模达 8.88 亿, 占网民整体的 $94.5 \%$, 其中短视频已 成为新闻报道新选择、电商平台新标配。网络新闻用 户规模为 7.25 亿, 占网民整体的 $77.1 \%$, 网络新闻 借助社交、短视频等平台, 通过可视化的方式提升传 播效能, 助力抗疫宣传报道。

在当下信息化快速发展的时代, 知识的更新和传 播速度也在飞速增长, 教育方式也日渐趋于多元化。 培养创新型人才是现代化教育所追寻的目标, 人们意 识到教育的工作重心已经由教会学习者知识转变为 教会学习者塑造健全人格。美术教育作为基础教育的 核心课程之一备受关注, 在这个知识社会和网络时 代, 美术课程的改革和进步必须是基于信息技术和面 向素质教育的。传统课程模式的局限性日益突出, 短 视频融入教学为我国中学美术鉴赏课程提供了新视 角和新思路, 探究短视频应用于在线教学的新模式, 为有效实施在线教学提供参考。

\section{2. 短视频应用于高中美术鉴赏教学中的特点}

从 2006 年微视频的概念诞生, 2013 年至 2016 年短视频从产生再到被定义为以 “秒” 为单位实现的 即时传播。短视频依靠其短小、灵活的特点, 切合了 用户的碎片化阅读习惯, 满足了大众个性化的需求。 本章节的主要目的在于分析短视频教学模式的特点, 短视频教学的出现适应了 “互联网+教育模式” 下教 育教学的新形式, 表现出传播模式创新, 教学资源丰 富这类特征。

\section{1. 传播模式创新, 提高知识传播效率}

短视频教学是符合当下信息化时代的一种教学 模式。短视频因其短小精悍的特点使其对视频的拍摄 技巧以及设备的要求都无需达到专业级水平, 短视频 制作低门槛化适应了用户碎片化的媒介接触习惯。目 前大多数短视频平台设置有创作者学院, 拍摄、音乐 特效等功能, 操作简单易学, 满足用户随拍随传的需
求。 1 便捷化的操作提高了用户创作的主动性, 促使 形成用户即是传播者, 也是创作者的创新传播模式。

在短视频教学模式下, 教师和学生同为知识的传 播者。在制作短视频的内容时可以亲自拍摄教学素 材, 不仅局限于对优秀的、多元化的内容进行拼接。 教师可以课程标准为参考, 选择适合学龄段的短视频 作为教学内容。同时, 学生可以将自己手机拍摄的内 容美化、加以配乐等步骤进行编辑制作后进行上传分 享, 制作出内容生动形象, 形式简洁明了的短视频。 促进学生学习的主动性, 一台手机就可以将学生纳入 知识传播者的范畴, 满足师生之间的互动参与感。2短 视频传播模式的创新, 让学生在担任知识的接收者的 同时也是知识的传播者, 同时满足知识随拍随传的需 求, 提高了知识传播的效率。

\section{2. 教学资源丰富, 有助于个性化学习}

“因材施教” 是孔子在其教育生涯实践中总结出 的一项重要的教学原则, 出自《论语. 先进篇》, 它 是指教师要从学生的实际情况出发、尊重学生的个别 差异, 对其进行有差别的教学, 使每个学生都能扬长 避短, 获得最佳发展。这一教育理念对于现代的教育 也具有重要的指导意义, 在传统班级授课制的课堂, 教师想要尊重学生的个性化差异非常困难。

在短视频教学模式下, 可以搜索到海量相关知识 量, 例如国画课程, 在课程外想要了解更多的用笔技 法、输入关键字即可检索到许多名师、国画家的示范 视频。即使是零基础的学生也可以通过详细的示范讲 解视频进一步了解国画。教师可以将示范过程编辑为 一个短视频合集，首先第一集演示基础的玉兰花画 法, 其次递进至对国画花鸟技法的演示分析, 最后延 展至对国画琵琶的解析。合集顺序的排列是根据难易 程度依次递进。分段式的教学演示视频有助于学生根 
据自身掌握情况进行相应的选择学习, 符合学生 个性化学习。(如图 1) 短视频教学丰富了教学内容, 拓宽了学生的视野。丰富的教学资源可以拓展学生对 于某一概念的认识和了解, 区别于传统教学方式里由 教师讲授的单一方式获得。同时, 内容创作者之间个 性化的差异也会使得同一主题呈现的方式多样化, 促 使教学资源更加丰富, 可供更多的老师们资源共享和 学生们的课外学习, 使得在新冠疫情这类特殊情况下 也能保障教学高效率。

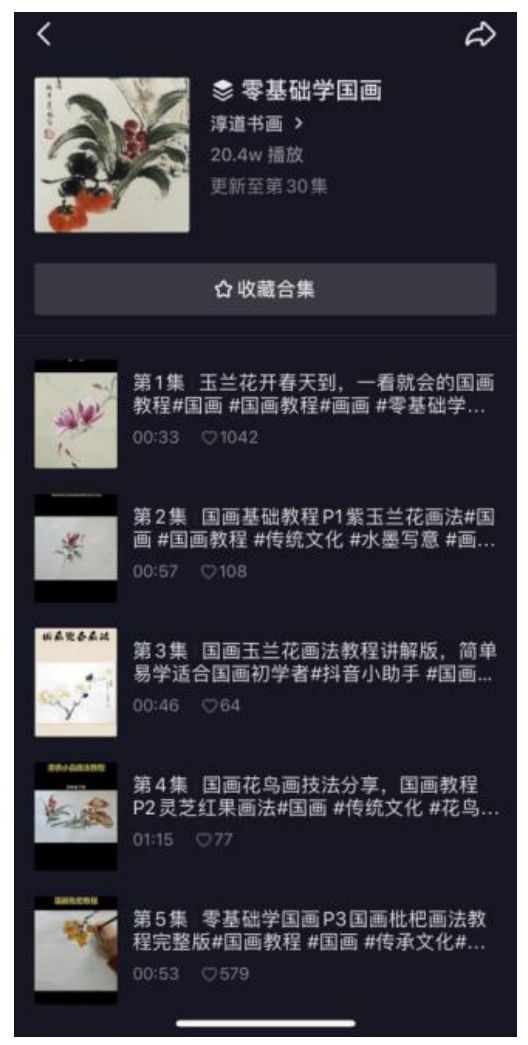

图 1 建立教学视频合集

\section{3. 短视频融入高中美术鉴赏课程之成效}

近年来，短视频在各种领域都有涉及，其中教育 领域中的短视频也得到了极大的发展。在新冠疫情的 影响下全国各校延缓开学, 教育部要求在防疫阶段实 行在线教学, 保证 “停课不停学” 。在此背景下, 短 视频教学模式迎来了机遇, 本研究意在将短视频教学 作为一种辅助型的在线教学新模式融入高中美术鉴 赏课程。利用短视频保障高中美术鉴赏在线教学质 量, 帮助学生拓宽艺术视角, 增强图像识图能力等。 改善传统的美术课堂环境, 促使学生对美术鉴赏课程 学习的积极性。提出短视频应用于在线教学的新模 式, 为有效实施在线教学提供参考。

\section{1. 鉴赏教材多元化，增强学生学习积极性}

传统美术鉴赏课堂在课堂学习部分可提供参考 教材案例多以达芬奇、蒙娜丽莎这类典型教材案例来
开展分析，千篇一律的教材知识容易造成教材趣味性 不足，会让学生对美术鉴赏的认识理解片面。选择的 素材多以美术教材为主, 很少利用网络资源。同时, 教材的更新速度慢, 在展示艺术作品的图像识图视角 有局限, 教材存在印刷图片的清晰度、饱和度、亮度 不足。教材里的部分文字表述内容跨度大、出现问题 过多、杂、跨度大, 部分文字表述过于理论, 不够接 地气, 不利于学生阅读。无法增强学生学习的兴趣性。

反观短视频教学模式下, 在鉴赏对象的选择上, 可以是民间技艺、国画、西方湿壁画，也可以是青花 瓷、工艺美术品、墙绘、官窑等。不局限于名画名家, 短视频平台收录有大量艺术创作者的创意作品。多元 的鉴赏对象才能有助于学生拓宽艺术视角, 掌握不同 的艺术鉴赏方法, 这样才能建立完善的美术教学体 系，体现鉴赏教学的真正效果。

\section{2 . 多角度展示艺术作品, 拓宽学生艺术视角}

在美术鉴赏教学中，教师要注重综合素养的培 养, 不能限制于让学生对画作进行鉴赏, 而是要引导 学生不断接受新鲜事物, 拓展自己的艺术视野。以《最 后的晚餐》课题为案例, 传统课堂的美术鉴赏课程在 新课导入部分多以 PPT 展示图片、播放相关视频导入。 习惯性地运用 “展示+讲解+总结” 的教学方式让学生 鉴赏名画。（见图 2) 首先, 图片展示的形式单一, 学生难以立即捕捉画面重点。容易导致高中美术鉴赏 课中学生的参与程度不高。3同时, 按部就班的美术 教学模式, 很容易让学生倦急。其次, 在叙述方式上 大篇幅地利用文字讲解画面内容, 教师一味地讲授理 论知识, 缺乏让学生独立思考的机会, 容易导致学生 阅读疲劳，造成兴趣低下、沉闪的鉴赏氛围。这样的 传统教学模式具有普遍性, 不利于学生的个性化发 展。

反观短视频教学模式, 首先应用故事性的讲述方 式, 以《最后的晚餐》为例延伸至湿壁画的创作, 通 过展示生动形象的湿壁画绘制画面, 拓展学生多元化 的艺术知识。（见图 3) 其次多角度展示原画作细节, 同时插入亲临现场鉴赏的画面, 结合字幕、背景音频, 渲染浓郁的课堂艺术气氛, 激发学生的想象力和创造 力。引导学生身临其境感受名画。（见图 4) 同时, 短视频教学实现了知识的互动传播, 每个视频的评论 区里都汇聚了观赏者们的想法, 在此进行交流, 建立 具有相同审美爱好的群体社交。（见图 5) 由此可见, 短视频教学有助于学生在广阔的美术世界中感受美、 认识美、发现美, 能够从多个层面体会作品中的价值, 领略艺术的鬼米力。 


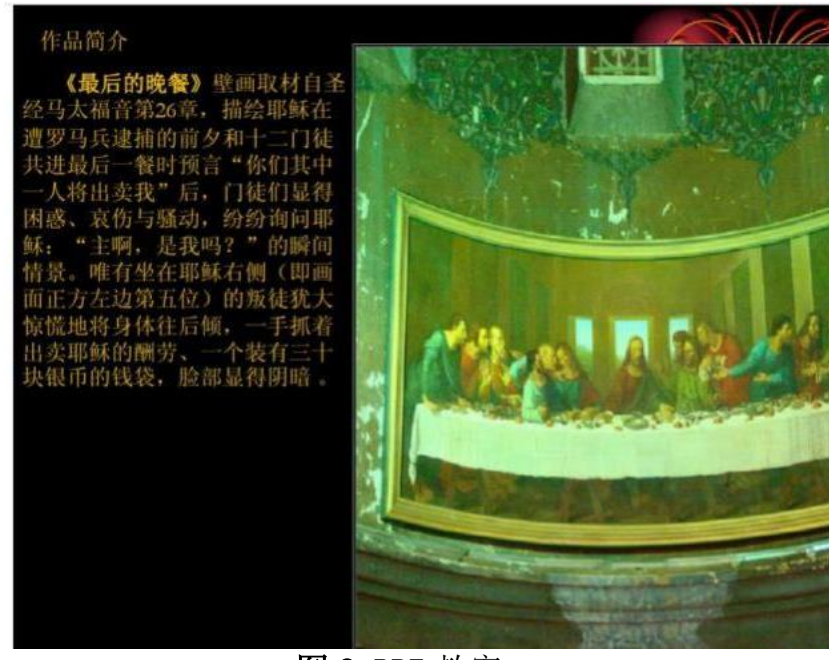

图 2 PPT 教案

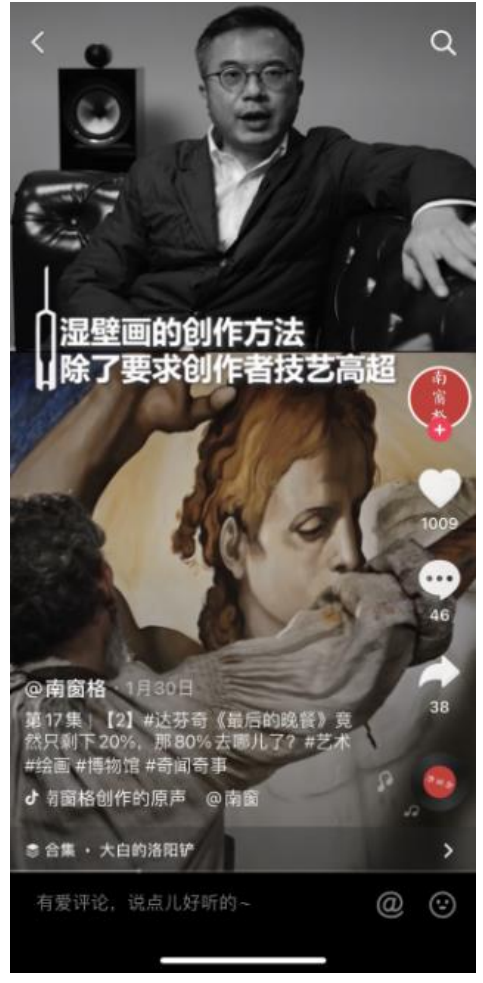

图 3 湿壁画创作

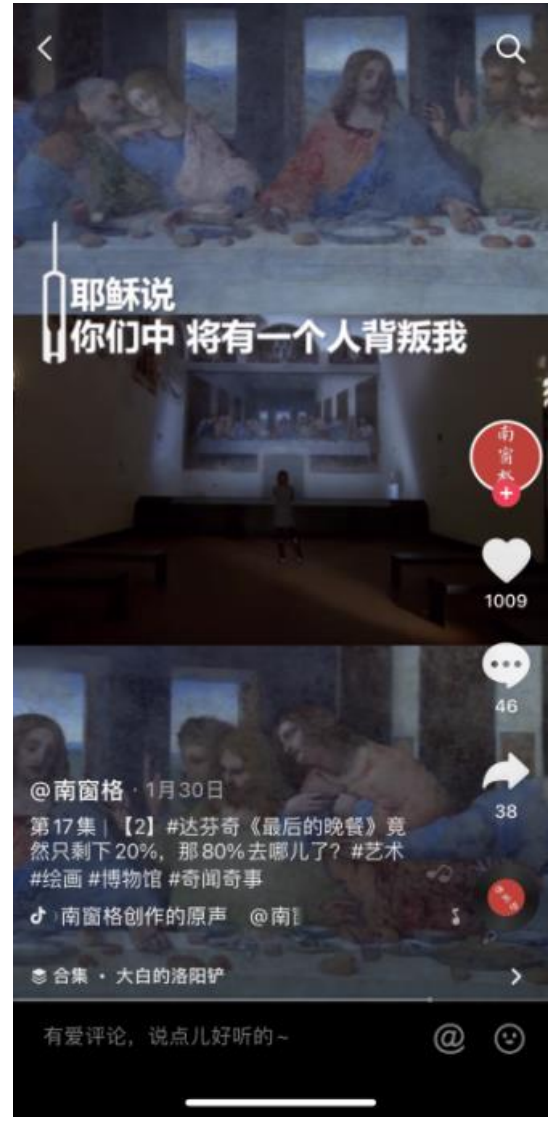

图 4 原画细节展示

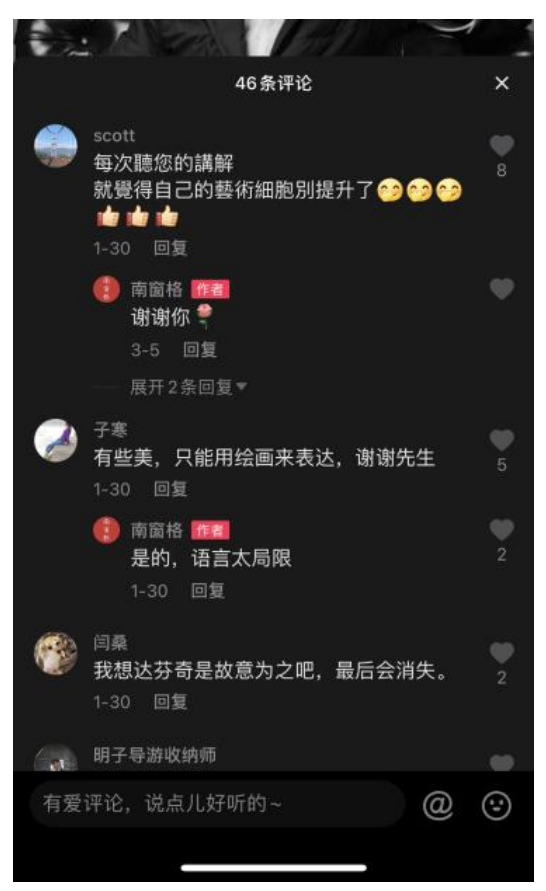

图 5 短视频评论区 


\section{3. 直观体验艺术情感，增强图像识图能 力}

图像识读是美术学科核心素养的组成内容之一, 也是学生进行有效美术鉴赏的首要前提。首都师范大 学尹少淳教授认为, 图像识读从审美心理角度来理 解, 其过程包含了三个层次。第一层次是观看, 学生 对作品的初步认识往往从视觉感官出发, 短视频教学 在展示艺术作品《千里江山图》时, 利用动静结合, 在画面的下半部分展示原画, 上半部分则是在原画的 基础上插入仙鹤、船只等动态运动结合主题音乐来演 绎 “会动的千里江山图”。将对应的字幕和音乐相结 合, 使得学生在视觉、感官上双重体验。学生能够根 据审美经验快速识读眼前的作品呈现的形式要素, 表 达出对作品的第一视觉感受。第二层次是识别, 短视 频教学的作品呈现方式, 通过镜头的运动, 学生能够 欣赏到画面的细节, 有助于学生识读作品的色彩、材 质等形式特征。第三层次是解读, 短视频教学结合了 音频搭配字幕的形式讲解艺术作品, 深入解析画面, 推动学生展开探究作品背后的象征意义和蕴藏的内 涵。在直观体验艺术情感中鉴赏名画, 短视频教学模 式有助于推动学生完成图像识读的过程, 形成视觉思 维, 增强图像识读能力。（见图 7)

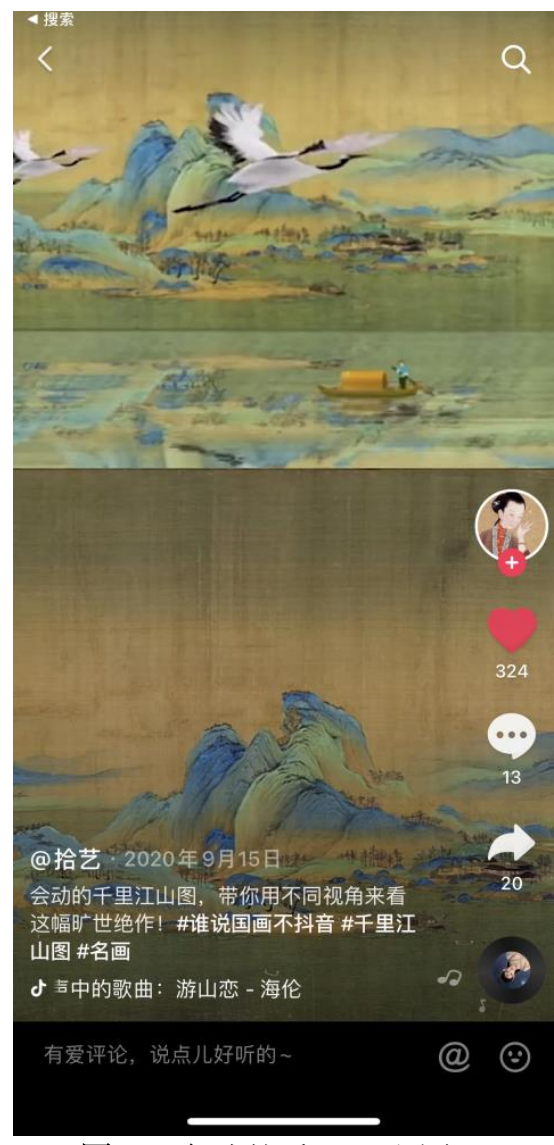

图 6《会动的千里江山图》

在现行的教育环境下存在的相关教学工具其实 有非常多种, 将短视频与传统教学模式有机地结合, 相辅相成, 共同促进教学的发展。传统的教学模式中,
老师是知识的传授者, 而学生则处于一种被动地学习 状态, 不利于培养学生的自主学习能力和全面发展。 在互联网+教育模式下, 短视频教学模式作为互助教 学工具, 极大地改变传统课堂单一的教学模式。为现 代化教育提供了丰富的优质资源共享, 使得人们对教 育观念有了新的认识, 有效促进了学生们个性化的发 展。

\section{4. 短视频融入高中美术鉴赏课堂 “三步走”}

基于对短视频教学模式融入高中美术鉴赏课程 中的研究和分析, 笔者根据新课改要求以保障线上教 学质量为主体在此设计了短视频教学模式下的 “三步 走” 教学实施计划。

\section{1. 精准教学活动}

高中美术鉴赏教学的目的在于培养学生的美术 鉴赏能力, 通过对艺术作品的赏析获得审美愉悦和知 识, 形成审美心理结构, 学会美术鉴赏的方法, 并激 发学生的创造力, 提高学生的动手实践能力, 促进学 生审美心理结构和鉴赏方式的完善。

这就要求我们在组织教学活动时注重学生审美 心理结构的完善和学会美术鉴赏方式。短视频教学模 式下可以以开展 “线上美术作品展” 活动, 利用短视 频能够随时随地观看的优势, 提高学生学习的主体意 识。不同于传统的美术展览, 需要布置展览现场, 需 要应对学校的具体条件、老师的策划能力、材料准备 等问题。展览的方式由 “现场展示” 转变为 “线上展 示”，不限制展览的规模大小，线上美术展览是在线 上鉴赏课程结束后，引导学生在日常完成作品时录制 短视频, 这类作品视频不单包含一件作品, 可以以年 度、学期为单位, 制作作品合集短视频。展示的内容 不仅是作品的完成形态，可以从艺术构思、准备材料、 制作过程等多方面入手。

“线上美术作品展”不仅可以给学生们带来学习 的热情, 更重要的是可以培养学生的主体意识, 让学 生主动地去学习, 落实以 “学生为本” 的教育观, 将 学生放在学习的主导位置。

开展 “线上美术作品展”一样具有教育功能, 鉴 赏作品的人群不单是班级的学生和老师, 还有每一位 热爱艺术的观看者。收获的每一个点赞都是通过互相 交流给予的肯定, 使得作品展成为一种文化资源, 在 大数据的时代, 良好的美术评价方式可以体现为评论 区的一句点评、随手的点赞 + 关注, 都是交流和认识 的反馈。

\section{2. 专业教学方法}

\section{2. 1. 建立互动性学习环节}

在短视频教学模式下, 建立互动性教学环节体现 在利用短视频平台中的留言区或者评论区等互动版 
块。保证在直播课堂结束后, 老师也能快速地看到学 生的问题并及时作答, 解决学生的疑惑同时了解其学 习和掌握程度。不仅增加了师生之间的互动, 还有助 于同学之间、观赏者之间的互动。当教师发布一条美 术鉴赏视频, 学生提出疑问, 为其解答的可以是其余 同学、老师或者是某一位艺术爱好者。评价方式更加 多元化, 从评价主体来看, 包括小组评价、个人评价、 老师评价; 学生将会收获更丰富、多样的回答。不同 的想法汇聚在评论区内, 为学生提供了更多文化资源 以及交流学习的机会。

\subsection{2. 碎片化课程, 促进重难点学习和记忆}

互联网腾飞的时代背景下涌现出的丰富技术及 工具促进了学习环境碎片化, 为快节奏生活的人们提 供了丰富学习环境。它消除了学习时空限制, 通过整 合提供了数字化资源环境和广阔学习空间。

经典的线上课程学习课程最突出的缺点在于脱 离了传统课堂上老师对学生的监管, 学生的注意力容 易分散, 过长的视频的观看不利于学生对知识的理 解, 最终呈现的教学效果差。鉴于经典线上教学模式 的局限性, 教师可以利用短视频 “短、平、快” 的特 点, 将完整的一套教学内容分为多段短视频来呈现, 每一段对应一个知识点, 而多个知识点就汇聚成一张 知识网或者是我们熟知的易读易懂的思维导图。有利 于学生对知识的重难点部分重点学习, 短视频的普及 性和便捷性使得学生可以随时随地观看, 进行重复学 习。4短视频比传统的文字、图片来得更加直观, 碎 片化的学习可以更迅速、便捷地获取知识。短视频符 合人们碎片化的阅读习惯, 随时随地可以观看, 一定 程度上还避免了学生因疫情暴发而无法学习的情况。 保障在新冠疫情这类突发性紧急情况出现时高中美 术鉴赏课程的正常进行。

\section{3. 重组教学流程}

短视频教学模式在初中美术鉴赏学习领域的教 学模式形成离不开课前准备阶段、课中应用分析阶 段、课后综合评价阶段这三个阶段的组成。

\section{3. 1. 课前}

许多问题在短视频教学模式下, 教师在确立教学 目标后, 可以借助互联网收集相关教学资源, 参考教 学资料, 自制或引用相关视频。建立新课的视频合集, 安排学生在课程开始前进行学习、讨论、思考, 为学 生自主获得信息资料提供便捷的平台。预习是学生掌 握新知识之前的了解其概要, 做到心中有数。由于当 代中学生的功课压力大, 所以学生在每次上课前, 未 必有时间预习功课。而短视频短小精悍的功能使得学 生只要善于利用每一分钟, 例如利用的时间段是早晨 上学途中、等车时间等。因为时间极为有限, 有时反
而会有意想不到的效果。

\section{3. 2. 课中}

首先确立问题, 在短视频平台进行直播授课结束 之后, 教师可以对课题进行小结, 分析总结学生反馈 的问题。再同学生进行沟通和讨论, 调动每个学生的 积极性。其次自主探索, 自主探究学习是学生必备的 一种学习方式, 包括自主认知、思考、经营。先由学 生对课程规定的视频合集进行自主欣赏。并引导学生 在观察的过程中对鉴赏对象进行分析。在直播课中, 讲解和归纳知识点, 使学生掌握一定的鉴赏方法。最 后组织小组合作学习, 在学生经过自主探究学习之 后, 学生可进行小组讨论, 对学习过程中遇到的问题 展开探讨, 让每位学生都积极参与到课堂中来。

\section{3. 3. 课后}

课后阶段也是美术鉴赏课程的重要环节, 根据课 堂讲授的内容布置课后作业, 让学生按时发布视频作 业。在发布视频的同时, 引导学生汶览相关鉴赏知识 视频。观看之后对自己的作品进行自评, 在评论区置 顶自己的自评内容, 培养学生自我评价的习惯。对自 己的上课表现和作品的创作表现进行自我评估。

\section{5. 结论}

随着国家教育体制改革的不断深化和发展, 单一 的课堂教学模式已无法切合教育的需求。新冠疫情背 景下, 教育部号召采用 “互联网+教育模式” , 在此 背景下, 将短视频应用于在线教学的新模式是顺应时 代发展, 是教学发展的创新模式。利用现代技术, 将 艺术与科技相结合。提高学生学习兴趣, 作为新的媒 介介入活跃课堂氛围。作为互助教学工具, 在线下可 以与传统教学模式相辅相成, 弥补传统课堂存在的教 学形式单一等问题, 教师可以将短视频教学模式与传 统教学模式相结合, 实现彼此之间的优势互补。在线 上, 短视频可以加入在线教学平台的队伍, 完善在线 教学的模式, 提高高中美术鉴赏课程在线教学的质 量, 共同促进教学的发展。科学合理地将短视频教学 模式应用在高中美术鉴赏课堂的教学中, 促进学生表 现美、发现美、欣赏美的水平的提高, 这是当前我国 从事教育研究工作者要考虑的重要问题。

\section{REFERENCES}

[1] Tian Bing.(2018).Research on content production and communication mode of online short video applications.http://cdmd.cnki.com.cn/Article/CDM D-11832-1018009021.htm.

[2] Wang Jing.(2016).Research on the application of the Flipped Class Model in art teaching of junior high school.https://d.wanfangdata.com.cn/thesis/D00992 685 . 
[3] Zhang Ying.(2017).The application of teaching resources micro-video in the art appreciation class of junior high school. https://cdmd.cnki.com.cn/Article/CDMD-11658-10 17227716.htm.

[4] Wang, J.J.(2019). Innovative practice of art appreciation teaching in junior high schools under the background of new curriculum reform. Research on Art Education,16:128-129.

[5] Chu, J.(2019).Cultivating students' key competences through art appreciation teaching in junior high school. Research on Art Education,18:148-149.

[6] Zhao,C,H. Wang,Y,D.(2019).The characteristics of short video teaching "Tik Tok" and its implications for vocational education. Mechanical Vocational Education,04:43-45.DOI:001:10.16309/j.cnki.issn. 1007-1776. 2019. 04. 013.

[7] Mu,R. Fu,Y.(2020).Exploration and investigation of online teaching practice under the epidemic Covid-19. Health Vocational Education, 17:127-128.

[8] Wang Mi.(2013). Content design of micro-video courses towards the era of fragmented learning. http://cdmd.cnki.com.cn/Article/CDMD-10269-10 14150412.htm

[9] Pang,L,J. Wang,R,X. Li,J,C.(2020).Research on the application of Flipped Class in physical education under the epidemic of Covid-19. High Education Journal, 17:105-111.

[10] Qian,P,H.(2018).Improve students' image reading ability. Jiangsu Education,49.

[11] Zhou Fei Qiao.(2016).Research on the communication mode and strategy of short video applications in the Internet era. https://www.docin.com/p-2094691613.html. 